\title{
L'APPROXIMATION DU CHAMP CRISTALLIN
}

\author{
Par J. M. WINTER, \\ Service de Physique du Solide et de Résonance Magnétique, Centre d’Études Nucléaires de Saclay.
}

Résumé. - Dans cet article on utilise l'hypothèse du champ cristallin pour expliquer les propriétés des ions du groupe du fer placés dans un champ cubique. En particulier, on détermine dans quelle situation le moment orbital est bloqué. Les propriétés des ions du groupe des terres rares sont brièvement discutées. Enfin on s'intéresse à la détermination des paramètres du champ cristallin.

Abstract. - In this article the crystal field theory is applied to explain the ground state properties of ions of the iron group in a cubic environment. We determine in what situation the orbital momentum is quenched. We also very briefly review some properties of the rare-earth ions. The evaluation of the crystal field parameters is discussed.

Dans le précédent article [1] nous avons expliqué comment le calcul de la structure du niveau fondamental d'un ion magnétique dans un cristal devait être mené. Ces considérations vont être maintenant appliquées à un certain nombre d'exemples. Un des plus intéressants et aussi des plus étudiés est le cas du groupe du fer.

I. Les ions du groupe du fer placés dans un champ cubique. - A) Générai ITÉs. - Ces ions ont des configurations du type $(3 d)^{n}$ ( $n$ étant compris entre 1 et 9). Nous allons, pour le moment, supposer que la symétrie du champ cristallin est cubique. La valeur du potentiel cristallin est en général supérieure à la valeur du couplage spin-orbite, mais inférieure à la distance en énergie entre termes. En pratique, les symétries rencontrées ne sont pas toujours cubiques, mais si l'on écrit le potentiel cristallin sous la torme:

$$
V=K+v
$$

où $K$ a la symétrie cubique et $v$ une symétrie moindre, il se trouve qu'en pratique $K \gg \lambda$ alors qu'en général $v$ est du même ordre de grandeur que $\lambda$.

La marche des calculs sera alors la suivante : on part du terme fondamental, l'action du champ cubique $K$ est étudiée. On s'intéresse particulièrement à déterminer si l'état fondamental ainsi obtenu garde ou non une dégénérescence orbitale. Ensuite, on étudiera l'effet sur ces états de $v$ et du couplage spinorbite. Dans le tableau 1 on trouvera la liste des termes fondamentaux ainsi que les valeurs de $\lambda$ déduites des spectres atomiques.
On remarque de suite que les seuls états orbitaux qui interviennent sont des états $S(L=0), D(L=2)$, $F(L=3)$. Pour l'état $S$ il n'y a déjà plus de dégénérescence orbitale, le magnétisme ne peut qu'être dû au spin seul. Il nous faut étudier l'action du champ cubique sur les niveaux $D$ (dégénérescence 5 ) et $F$ (dégénérescence 7). La réponse à cette question nous est immédiatement fournie par la théorie des groupes [2], [3]. En ce qui concerne les niveaux $D$, les résultats se trouvent à l'aide d'arguments assez simples.

Considérons donc les parties angulaires des fonctions d'onde correspondant à un niveau $D$, une fonction possible est $x y_{\mid} r^{2}$ (exprimée en fonction des harmoniques sphériques), elle s'écrira

$$
\frac{x y}{r^{2}} \simeq \frac{Y_{2}^{2}(\theta, \varphi)-Y_{2}^{-2}(\theta, \varphi)}{l} .
$$

En permutant les axes on forme deux autres fonctions $y z$ et $z x$ orthogonales à la première. La symétrie cubique exige que les trois niveaux correspondant à ces trois fonctions soient dégénérés. Nous aurons donc un triplet.

De la même façon les trois fonctions

$$
\frac{x^{2}-y^{2}}{r^{2}}, \quad \frac{y^{2}-z^{2}}{r^{2}}, \quad \frac{z^{2}-x^{2}}{r^{2}}
$$

correspondent à des niveaux dégénérés. Ces trois fonctions ne sont pas indépendantes et se réduisent à deux, par exemple $x^{2}-y^{2}$ et $2 z^{2}-x^{2}-y^{2}$. Nous avons un doublet. Il existe malheureusement un grand

\begin{tabular}{|c|c|c|c|c|c|c|c|c|}
\hline$(3 d)^{1}$ & $(3 d)^{2}$ & $(3 d)^{3}$ & $(3 d)^{4}$ & $(3 d)^{\mathbf{5}}$ & $(3 d)^{6}$ & $(3 d)^{7}$ & $(3 d)^{8}$ & $(3 d)^{9}$ \\
\hline $\begin{array}{l}\mathrm{I}_{1}++++ \\
{ }^{2} D\end{array}$ & $\begin{array}{c}\mathrm{V}+++ \\
{ }^{3} F\end{array}$ & $\begin{array}{c}\mathrm{V}++, \underset{4}{\mathrm{Cr}}+++ \\
{ }^{F}\end{array}$ & $\begin{array}{c}\mathrm{Cr}++ \\
{ }^{5} \mathrm{D}\end{array}$ & $\begin{array}{c}\mathrm{Mn}^{++}, \mathrm{Fe}^{+++} \\
{ }^{6} S\end{array}$ & $\begin{array}{c}\mathrm{Fe}^{++} \\
{ }^{5} \mathrm{D}\end{array}$ & $\begin{array}{c}\mathrm{Co}^{++}+ \\
{ }^{4} F\end{array}$ & $\begin{array}{c}\mathrm{Ni}++ \\
{ }^{3} F\end{array}$ & $\begin{array}{c}\mathrm{Cu}++ \\
{ }^{2} D\end{array}$ \\
\hline $4 \mathrm{~cm}^{-1}$ & $104 \mathrm{~cm}^{-1}$ & $54 \mathrm{~cm}^{-1}, 87 \mathrm{~cm}^{-1}$ & $57 \mathrm{~cm}^{-1}$ & & $-100 \mathrm{~cm}^{-2}$ & $-180 \mathrm{~cm}^{-1}$ & $-355 \mathrm{~cm}^{-1}$ & $-852 \mathrm{~cm}^{-1}$ \\
\hline$\Gamma_{5}$ & $\Gamma_{4}$ & $\Gamma_{2}$ & $\Gamma_{3}$ & $\Gamma_{1}$ & $\Gamma_{5}$ & $\Gamma_{4}$ & $\Gamma_{2}$ & $\Gamma_{3}$ \\
\hline
\end{tabular}
nombre de notations pour ce triplet et ce doublet.

\section{TABLEAU 1}

La ligne 1 donne la configuration. La ligne 2 donne des exemples d'ions ayant une certaine configuration. On trouve ensuite la liste des termes fondamentaux, puis les valeurs du couplage spin-orbite. Enfin dans la dernière ligne on indique l'état fondamental pour un environnement octaédrique régulier. 


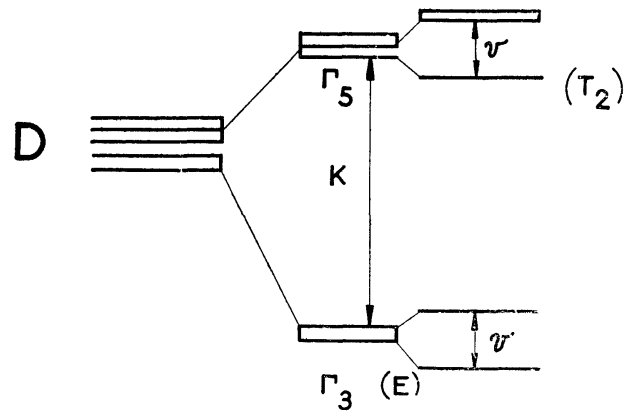

Terme + champ cubique + champ non cublque

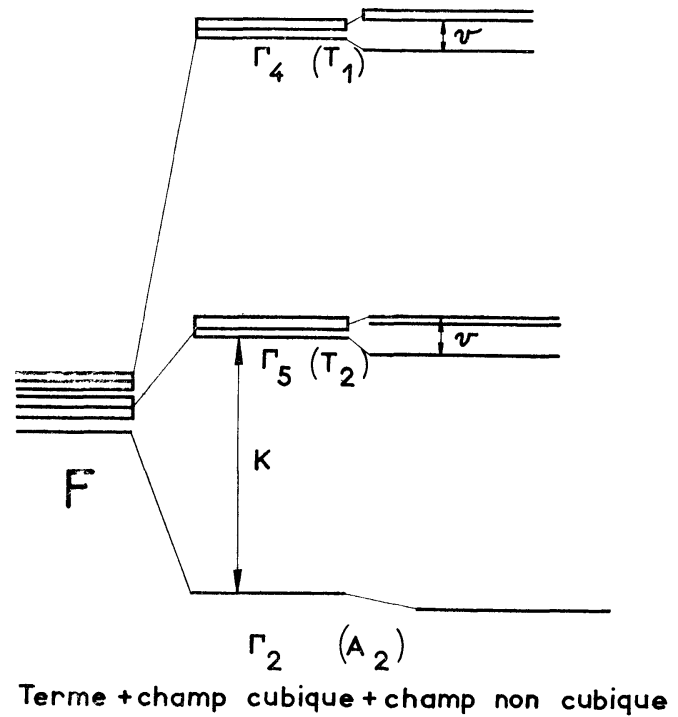

Fig. 1. - Séparation des niveaux orbitaux $D$ et $F$ par le champ cristallin.

Nous utiliserons celle de Bethe [2] (nous ajouterons entre parenthèses celle de Mulliken [3]). Le triplet se dénomme $\Gamma_{5}\left(T_{2}\right)$ et le doublet $\Gamma_{3}(E)$. De la même façon (ou bien en utilisant la théorie des groupes) on montre que le niveau $F$ se décompose en 2 triplets et 1 singulet (qui se comporte du point de vue angulaire comme $\left.x y z / r^{3}\right)$.

Comme nous l'avons déjà fait remarquer, la valeur du champ cubique pour des électrons $d$ ne dépend que d'un paramètre. Ceci a pour conséquence que la position relative des trois niveaux est toujours la même. (Notons que les deux triplets provenant de l'état $F$ ont des notations différ entes, les fonctions d'onde de ces niveaux ne se comportant pas de la même façon par rapport aux opérations laissant un cube invariant).

Enfin, avant d'abandonner ces considérations basées sur la symétrie, mentionnons une importante propriété des niveaux $\Gamma_{\mathbf{3}}$. On peut montrer que les valeurs moyennes des composantes de $\mathbf{L}$ sont nulles pour les niveaux de $\Gamma_{\mathbf{3}}$. Le calcul peut se faire en

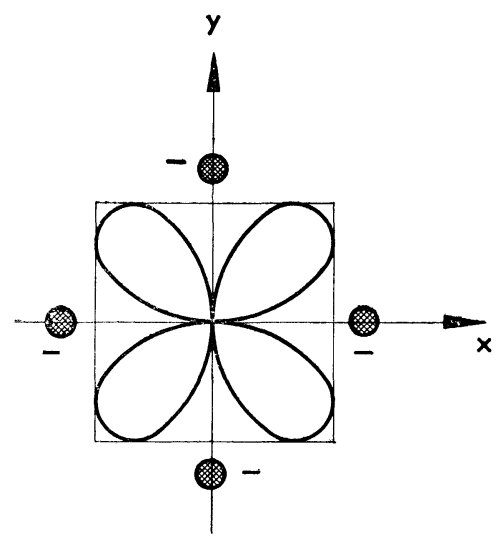

$$
\text { orbitale } x y / r^{2}
$$

FIG. 2. - Allure de la densité électronique dans le plan xOy pour les orbitales $x y$ et $x^{2}-y^{2}$. utilisant les parties angulaires des fonctions d'onde, ou bien par des considérations de théorie des groupes.

En résumé, si le niveau fondamental est un singulet $\Gamma_{2}$, ou bien un doublet $\Gamma_{3}$ (ou évidemment un état $S$ ), le magnétisme orbital sera bloqué. Maintenant nous allons déterminer le niveau d'énergie le plus bas.

B) DÉtermination DE L'ÉtAT Fondamentai . - La situation expérimentale la plus fréquente est celle d'un ion entouré d'un octaèdre régulier de molécules d'eau. Le champ cristallin peut être considéré comme le champ électrostatique des six ions oxygène. (On traiterait de la même façon le cas des six ions $\mathbf{F}^{-}$).

Cherchons à déterminer quel est dans cet environnement le (ou les) niveau(x) d'énergie minimale.

Prenons d'abord le cas d'un seul électron d. Les trois orbitales de $\Gamma_{5}$ ont des densités électroniques nulles dans les plans 100,010 et 001 et maximales dans les plans bissecteurs de ces plans. Donc les électrons évitent les charges placées le long des axes (voir fig. 2)

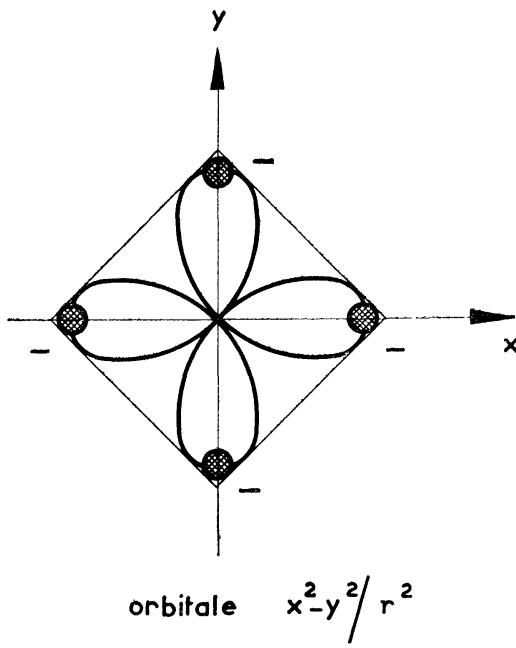


Au contraire, les fonctions d'onde correspondant aux niveaux $\Gamma_{3}$ ont des densités électroniques maximales le long des axes. A cause de la répulsion coulombienne, le triplet aura une énergie inférieure. Ceci résout le problème pour l'ion $\mathrm{Ti}+++$. Pour déterminer les états fondamentaux des autres configurations nous allons utiliser une méthode due à Kotani [4]. Considérons les 5 niveaux possibles pour un électron $d$ dans un champ cubique, séparés en triplet et doublet. Nous allons placer les $n$ électrons sur ces niveaux et chercher le niveau d'énergie le plus bas, cnmpatible avec le principe de Pauli et la règle de Hund pour le spin. Étudions par exemple $(3 d)^{2}$ (nous savons que le terme fondamental est ${ }^{3} F$ ) $S=1$. Nous formerons donc un état $m_{s}=1$ en fixant les $m_{s_{1}}$ et $m_{s_{\mathbf{v}}}=1 / 2$ (fig. 3). Dans ces conditions l'état d'énergie le plus bas aura un électron dans un des niveaux de $\Gamma_{5}$, l'autre dans un autre niveau de $\Gamma_{5}$ (pas le même à cause du principe de Pauli). Cet état a visiblement une dégénérescence orbitale d'ordre 3 . Il y a en effet trois possibilités pour le trou dans $\Gamma_{5}$. On peut montrer que cet état est en réalité ${ }^{3} \Gamma_{4}$. F́tudions $(3 d)^{3}$ de même, pour un état $m_{s}=3 / 2$ les trois orbitales sont occupées. Il n'y a plus de dégénérescence orbitale ; nous avons ${ }^{4} \Gamma_{2}$. Dans le tableau 1 nous donnons l'état d'énergie le plus bas pour l'environnement octaédrique.

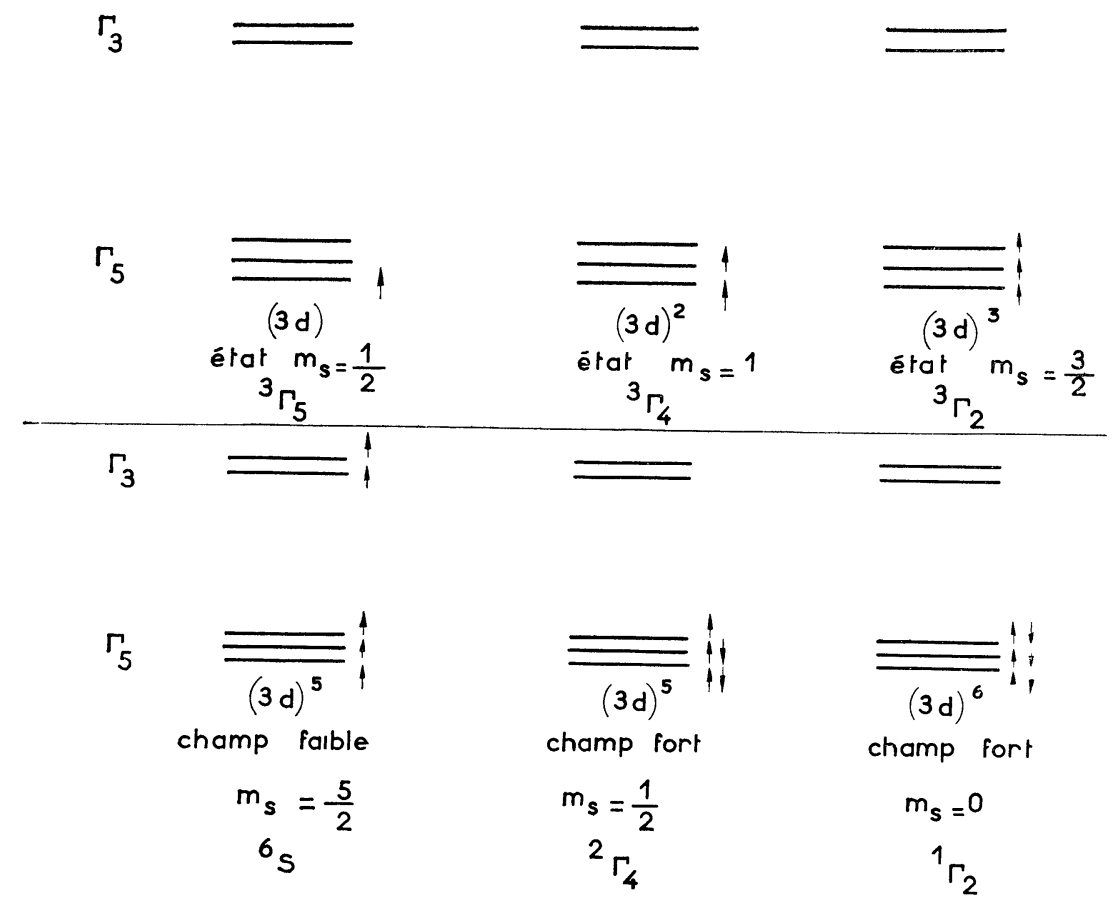

Fig. 3. - Détermination de l'état fondamental pour les configurations $(3 d)^{n}$ dans un champ cubique, en considérant les niveaux d'énergie à 1 électron. Les deux états de spin sont repérés par l'orientation de la flèche.

Dans la suite de la discussion on séparera donc les ıons en trois catégories : d'abord l'état $S(3 d)^{\mathbf{5}}$ où il n'y a en principe pas de problème (en réalité la précision des mesures de résonance permet dans ce cas d'observer des effets très faibles obtenus par des calculs de perturbation d'ordre élevé, nous n'en parlerons pas) ; ensuite les ions dont l'état fondamental est un singulet $\Gamma_{2}$ ou un doublet $\Gamma_{3}$ (le moment orbital est bloqué); enfin ceux dont l'état fondamental est un triplet (le moment orbital n'est pas bloqué).

C) Ions dont Le moment orbital est bloqué. Pour l'environnement octaédrique on rencontre cette situation pour les configurations $(3 d)^{3},(3 d)^{4},(3 d)^{8}$ et $(3 d)^{9}$. Pour les niveaux dont le singulet est le niveau fondamental, seul subsiste la dégénérescence de spin. Pour le doublet la dégénérescence est en général levée par les composantes non cubiques $v$ du champ cristallin et l'un des deux niveaux devient fondamental.

Dans les deux cas, le couplage spin-orbite est sans effet car les valeurs moyennes de $\mathbf{L}$ sont nulles. Il est toutefois souvent nécessaire de pousser les calculs plus loin.

Le couplage spin-orbite couple l'état fondamental aux états excités. L'état fondamental comporte une fraction de l'ordre de $\lambda / K$ d'états excités du genre triplet. Ceci a deux conséquences. Une partie de la dégénérescence de spin est levée même en l'absence d'un champ magnétique extérieur, les séparations sont de l'ordre de $\lambda^{2} / K$ (soit environ $1 \mathrm{~cm}^{-1}$ ) (cet effet est appelé structure fine) [5]. D'une façon assez grossière on peut dire que le spin entraine avec lui une portion $\lambda / K$ de moment orbital, le blocage est imparfait. La séparation des niveaux dans un champ magnétique extérieur (et par conséquent la susceptibilité) n'est plus celle correspondant à un spin pur. Cet écart par rapport au spin pur est, en général, anisotrope et de l'ordre de $\lambda / K$. Dans le cas de l'ion $\mathrm{Cu}^{++}$, cette correction peut aller jusqu'à $20 \%$.

D) IONS DONT LE MOMENT ORBITAL N'EST PAS BLOQUÉ [6]. - Dans ce cas, il est beaucoup plus diffi- 
cile de faire des considérations générales. Partant du triplet orbital, il faut étudier simultanément l'action du couplage spin-orbite et des composantes $v$ du champ cristallin. Les propriétés des niveaux les plus bas obtenus ainsi dépendent beaucoup de la symétrie de $v$. Expérimentalement, les moments magnétiques mesurés sont intermédiaires entre ceux calculés avec le spin seul et ceux calculés avec le spin et l'orbite. Ceci provient de ce que l'état fondamental triplet ne contient que trois des niveaux orbitaux (au lieu des 5 ou 7 en l'absence du champ cubique).

La séparation des niveaux en fonction du champ extérieur conduit à des facteurs $g$ très différents de 2 . Le facteur $g$ est défini par la formule suivante :

$$
\Delta E=g \beta H_{0}
$$

où $\Delta E$ est l'écart des niveaux et $\beta$ le magnéton de Bohr. Pour $\mathrm{Co}^{++}(3 d)^{7}$ on observe des facteurs $g$ très anisotropes et pouvant aller jusqu'à 6 . Ils dépendent beaucoup du cristal étudié.

E) Cas D'un CHAMP CRISTAllin FORT [7]. - Ce cas peut se comprendre simplement en reprenant la méthode de Kotani. Dans la discussion précédente, les électrons étaient placés sur les orbites en tenant compte de la règle de Hund. Si le champ devient fort l'écart $\Gamma_{3}, \Gamma_{5}$ grandit et, du point de vue énergie, il devient plus avantageux de peupler $\Gamma_{5}$ avant d'appliquer la règle de Huna. Le problème ne se pose d'ailleurs qu'à partir de la configuration $(3 d)^{4}$. Considéı ons par exemple la configuration $(3 d)^{5}$. En champ faible, la règle de Hund prédit $S=5 / 2$ et les 5 orbitales sont occupées. On a un état $S$. En champ fort, on placera les 5 électrons dans $\Gamma_{5}$. Le principe de Pauli ne permet alors que $S=1 / 2$ et l'état fondamental est un triplet orbital.
Un autre exemple est fourni par la configuration $(3 d)^{6}$ qui est diamagnétique en champ fort.

II. Le groupe des terres rares. - Dans ce cas $J$ cst un bon nombre quantique. Le champ cristallin lève la dégénérescence du multiplet. Dans l'ensemble, les comportements sont plus variés et plus difficiles à calculer que pour les ions du groupe du fer. On rencontre des valeurs de $J$ très élevées. La symétrie de l'environnement est en générale basse et, comme il faut pousser le développement du champ cristallin jusqu'à $l=6$, il y a un grand nombre de coefficients.

Un théorème dû à Kramers [8] joue un rôle très important. Il dit que, pour tout système possédant un nombre impair d'électrons, tous les niveaux ont au moins une dégénéresoence d'ordre 2 en l'absence d'un champ magnétique extérieur. Ce théorème n'est évidemment pas limité aux ions de terres rares mais il est très utile ici car il permet de dire que, quelle que soit la symétrie, l'état fondamental des ions de terres rares à nombre d'électrons impairs est dégénéré. Le champ magnétique lèvera cette dégénérescence et ceci est une condition nécessaire pour pouvoir observer une résonance aux fréquences usuelles. Considérons par

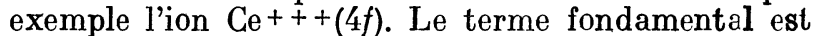
$S=1 / 2, L=3$, le couplage spin-orbite le sépare en deux multiplets, le multiplet $J=5 / 2$ étant le plus bas. Ce multiplet est dégénéré 6 fois. Le théorème de Kramers dit que la décomposition la plus complète conduira à trois doublets.

La symétrie est souvent trigonale et si on prend comme axe des $z$ l'axe trigonal, le nombre quantique $J_{z}$ demeure un bon nombre quantique et les trois doublets correspondent respectivement aux trois niveaux $J_{z}= \pm 1 / 2, J_{z}= \pm 3 / 2, J_{z}= \pm 5 / 2$. Il est alors facile de prévoir comment ces niveaux se séparent en

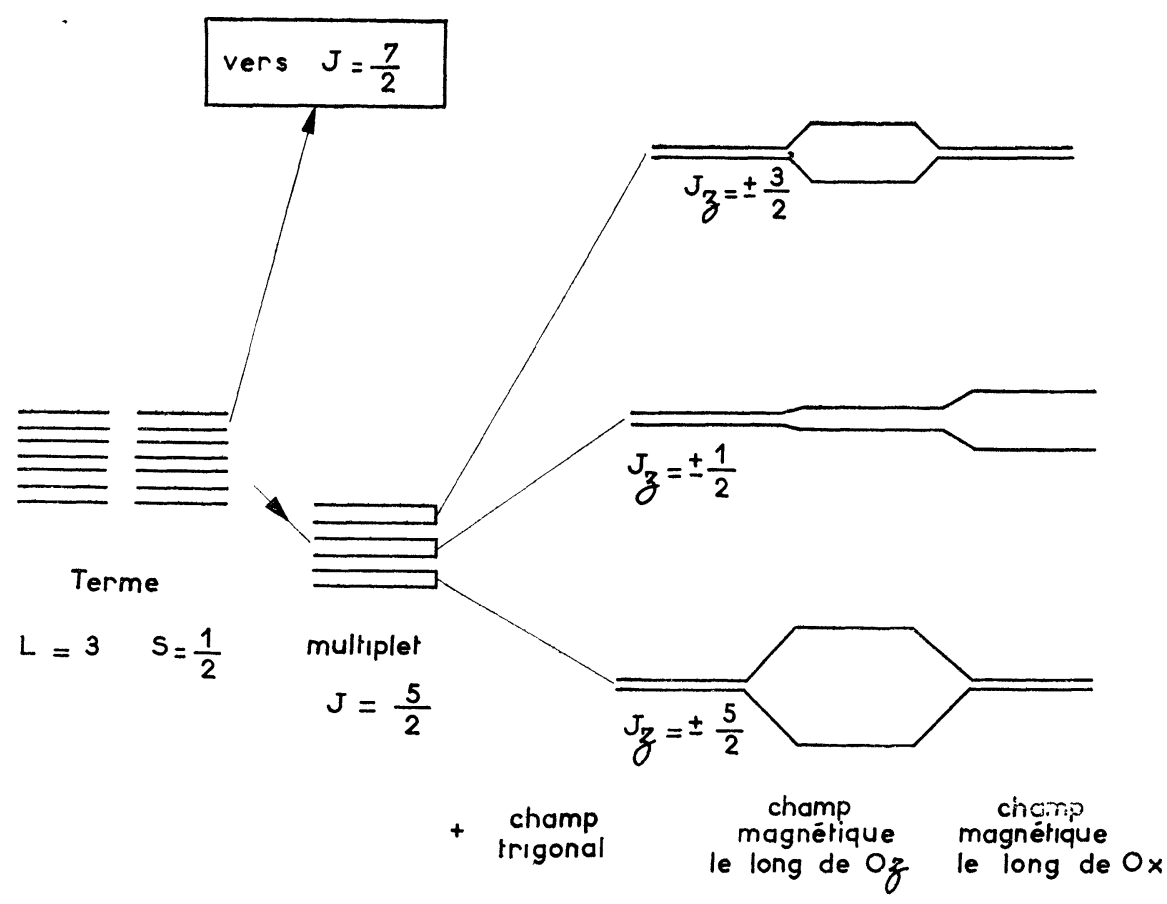

Fig. 4. - Niveaux d'énergie de l'ion $\mathrm{Ce}^{+++}+(3 f)$ dans un champ trigonal. On notera l'anisotropie de la séparation produite par le champ magnétique. 
présence d'un champ magnétique extérieur. Supposons que le niveau fondamental soit le doublet $\pm 5 / 2$, si le champ est parallèle à l'axe trigonal il donnera une séparation

$$
\Delta E=\left(m_{J}-m_{J}^{\prime}\right) g_{J} \beta H
$$

où $g_{J}$ est le facteur de Landé atomique pour le multiplet $J$

$$
m_{J}=-m_{J}^{\prime}=5 / 2
$$

$\Delta E=5 g_{J} \beta H$ si on écrit symboliquement cette séparation

$$
\Delta E=g_{\|} \beta H \quad g_{\|}=4,28 .
$$

Par contre si $H_{0}$ est perpendiculaire à l'axe trigonal, les niveaux ne sont plus séparés $g_{\perp}=0$.

De la même façon, on montrerait que les susceptibilités sont anisotropes. (Si la mesure est faite à une température suffisamment basse pour que seul le doublet fondamental soit peuplé.)

III. L'évaluation du champ cristallin [9]. Conclusion. - Nous n'avons pas parlé de la détermination des paramètres du champ cristallin à partir de la connaissance de l'environnement de l'ion. Le premier calcul dû à Van Vleck [10] et Polder [11] consistait simplement à remplacer les ions voisins par des charges ponctuelles et à calculer le champ électrostatique ainsi produit. Assez étonnamment, les résultats étaient en bon accord avec l'expérience. Les tentatives d'amélioration ont rendu l'accord beaucoup moins bon, certains calculs conduisant même à des résultats ayant le mauvais signe. En réalité, si l'on veut faire un calcul rigoureux, en tenant compte des électrons des ions voisins, il faut calculer le champ cristallin d'une façon self-consistante, c'est-à-dire comme un potentiel moyen dû aux noyaux et électrons des ions voisins. Ce calcul se révèle très difficile et, très rapidement, on arrive à se demander si un calcul aussi compliqué, faisant intervenir les fonctions d'onde des électrons des ions voisins, est utile car, en fait, la séparation entre potentiel de Hartree, dû à l'ion central, et potentiel moyen, dû au voisin, est artificielle. Dans ces calculs, la simplicité initiale du modèle du champ cristallin est perdue et il vaudrait mieux partir directement d'un modèle covalent où les fonctions d'onde, au départ, sont des mélanges de celle de l'ion central et des voisins. Ces questions ne sont pas encore complètement clarifiées. On peut toutefois conclure en remarquant qu'il est possible d'expliquer presque entièrement l'énorme ensemble des résultats expérimentaux sur le groupe du fer et celui des terres rares à l'aide des quelques paramètres du champ cristallin et cette constatation est en elle-même une excellente justification de l'approximation du champ cristallin $\left({ }^{1}\right)$.

Manuscrit reçu le 21 decembre 1964.

\section{BIBLIOGRAPHIE}

[1] Winter (J. M.), J. Physique, 1965, 26, 41 .

[2] Bethe (H. A.), Ann. Physık, 1929, 3, 133.

[3] Mulliken (R. S.), J. Chem. Physucs, 1935, 3, 375.

[4] Kotani (M.), J. Phys. Soc., Japan, 1949, 4, 293.

[5] Abragam (A.) et Pryce (M. H. L.), Proc. Roy. Soc., 1951, A 205, 135.

[6] Abragam (A.) et Pryce (M. H. L.), Proc. Roy. Soc., 1951, A 206, 173.
[7] Van Vleck (J. H.), J. Chem. Physucs, 1935, 3, 807

[8] Kramers (H. A.), Proc. Amst. Acad. Sc., 1930, 33, 959.

[9] Anderson (P. W.), Solzd State Physics, 14, édité par Seitz et T'urnbull (Academic Press, p. 181 à 190).

[10] Van Vleck (J. H.), J. Chem. Physics, 1939, 7, 72.

[11] Polder (D.), Physica, 1942, 9,-709.

$1^{1}$ Cette mise au point a faıt l'objet d'un exposé lors du Colloque sur le Champ cristallin, organıse, sous le patronage ve la Socıéte Françaıse de Physique et de l'Assocıatıon Françaıse de Cristallographie, de Novembre 1964 à Fevrier 1965.

L'ensemble des exposés presentés au Colloque sera reunı dans un fascicule spécıal, édıté par le Journal de Physique. 